\title{
Schweiz - Europa: Die Grenzen der bilateralen Verträge
}

Mit dem freien Personen-, Güter-, Kapital- und Dienstleistungsverkehr leistet die Europäische Union einen wesentlichen Beitrag zur wirtschaftlichen Entwicklung unseres Kontinents. Ihr politisches Vorhaben ist zwar bei weitem noch nicht abgeschlossen, doch es führte zu einer Kultur des Dialogs und der Zusammenarbeit zwischen den Mitgliedstaaten. Daraus resultieren Stabilität und Wohlstand, wovon alle - auch die Schweizerinnen und Schweizer - profitieren, selbst wenn gewisse Kreise daran zweifeln.

Die Freihandelsabkommen von 1972 und die bilateralen Verträge von 2002 haben massgeblich zu dieser Entwicklung beigetragen. Die Schweizerinnen und Schweizer sind damit zufrieden und vertrauen auf diesen Prozess, der ihnen die Möglichkeit zu geben scheint, der Bürokratie in Brüssel zu entkommen und ihre demokratischen Rechte und ihre Unabhängigkeit zu erhalten. Der Bundesrat und das Parlament müssen regelmässig die Kompatibilität der schweizerischen Gesetzgebung mit den Richtlinien und Verordnungen der Europäischen Union sicherstellen, die von den Europäern ohne Schweizer Beteiligung beschlossen wurden.

Auf diese Weise wurde auch das Medizinalberufegesetz erarbeitet. Die gegenseitige Anerkennung von Ausbildungsabschlüssen mit den europäischen Staaten und der freie Personenverkehr - im vorliegenden Fall im Zusammenhang mit den Medizinalberufen - sind ein wesentlicher Bestandteil dieses Gesetzes. Doch seltsamerweise wurden für den Verordnungsentwurf nicht alle Richtlinien der EU zu diesem Bereich berücksichtigt. Dies gilt insbesondere für die Richtlinie 2005/36/EG. Diese legt die Kriterien für die gegenseitige Anerkennung der Spezialisierungen fest und sorgt so weit als möglich für eine Vereinheitlichung der Bezeichnungen.
Die verschiedenen nationalen Instanzen für die Validierung der Kompetenzen, die für die Vergabe der Berufsausübungsbewilligungen zuständig sind, sowie die entsprechenden nationalen Berufsverbände sind mehrmals zusammengekommen, um die erforderlichen Zertifizierungsstandards zu erarbeiten. Damit soll den Ärztinnen und Ärzten aus EU-Staaten die Möglichkeit gegeben werden, sich in einem anderen Mitgliedstaat niederzulassen und dort zu praktizieren. Das erste Treffen fand im Jahr 2005 in Edinburg unter dem Vorsitz von Grossbritannien statt. Die weiteren Zusammenkünfte erfolgten im Jahr 2006 in Helsinki und letzte Woche in Berlin. Im Gegensatz zum BAG nahm die FMH an diesen Treffen teil. Dabei ging es immerhin darum, unter Anwendung der Richtlinie 2005/36/EG einen europäischen Musterausweis zu erarbeiten.

Aus nur schwer verständlichen Gründen wird dieser gesamte Prozess im Verordnungsentwurf des BAG zum MedBG völlig ausser acht gelassen. Der Entwurf berücksichtigt die in der Richtlinie 1993/16/EG festgehaltenen Bestimmungen, die offensichtlich veraltet sind. Der Facharzttitel der Europäer wird anerkannt, so dass sie in der Schweiz praktizieren können. Doch die Schweizer können a priori nur mit Mühe vom verlangten europäischen Ausweis profitieren, um in einem EU-Land zu praktizieren. Und falls sie es trotzdem versuchen, werden sie zweifellos Schwierigkeiten mit der Bürokratie des Bundes und der Kantone bekommen. Nun sind wahrscheinlich neue Verhandlungen im Rahmen des gemischten Ausschusses Schweiz-EU erforderlich, um die Richtlinie 2005/36/EG zu berücksichtigen. Weshalb einfach, wenn's auch kompliziert geht ...?

Dr. med. Yves Guisan, Vizepräsident der FMH, Nationalrat 